\title{
A Study on Humanoid Robot Control Method Using Zigbee Wireless Servo Motor with Sensor Network
}

\author{
Dae Seob Shin*, Hyeongcheol Lee*
}

\begin{abstract}
In this study, we developed two legged multi-joint robot by using wireless servo motor that was applied by wireless sensor network technology, which is widely used recently, and performed an experiment of walking method of two legged multi-joint robot. We constructed the star network with servo motors which were used at each joint of two-legged robot. And we designed the robot for operation by transmission of joint control signal from main control system or by transmission of the status of each joint to the main control system, so it operates with continuously checking the status of joints at same time. We developed the humanoid robot by using wireless digital servo motor which is different from existing servo motor control system, and controlled it by transmitting the information of angles and speeds of robot joints to the motor(node) as a feedback through main control system after connecting power and setting up the IDs to each joint.

We solved noisy problem generated from wire and wire length to connection point of the control device by construction of the wireless network instead of using existing control method of wiring, and also solved problem of poor real time response to gait motion by controlling the position with continuous transmission of control signals to each joint. And we found that the effective control of robot is able by performing the simulation on walking motion in advance with the developed control algorithm which was downloaded into installed memory.

Also we performed the stable walking with two-legged robot by attaching pressure sensor to robot sole. And we examined the robot gait operated by application of calculated algorithm on robot movement to each joint.

In this study, we studied the method of controlling robot gait motion by using wireless servo motors and measured the torque applied to each joint, and found that the developed wireless servo motor by ZigBee sensor network offers easier control of two legged robot gait and better circuit configuration of it than the existing wired control system could do.
\end{abstract}

Key words: Actuator, Two-legged Robot, ZigBee, Sensor Network, Servo Motor

\section{Introduction}

The interest in robotics has been globally increased and diverse studies on robotics have been performed as well. Thus, the more advanced robots will be studied in the future. In particular, robots that are able to walk with legs are popularly under study[1,2,3,4]. Quadrupedal, hexapod or articulated robots have the structure of easier movement, though the study on human style gait of

*Department of Electrical and Biomedical Engineering, Hanyang University two-legged robot will be continued[1,2,3].

Generally the experiment on articulated robot like quadrupedal or hexapod robot can be safely and successfully performed from simulation level to physical aspect due to its many legs. But experiment on two-legged walking robot may end with unsatisfying result.

And noises generated from the wires which are used for each joint, servo motor, control unit to transmit the control signals and power may cause malfunctions, incorrect simulation results, or unstable operations. But servo actuator to which the technology of ZigBee wireless sensor network is applied[5] can offer efficient control on robot's walking motion. 
We developed the algorithm for two-legged robot gait with the control of wireless sensor network, and verified that the simulated motion by experiment can be effectively applied to the actual robots.

Main control system transmits the signals on angle and speed of each moving joint. And it controls the status of each joint by checking feedback signals on joint status according to joint motion of robot. Therefore immediate action on walking motion of robot in case robot joint troubles can be ensured.

And stable gait was also ensured with attachment of the pressure sensor to the sole of robot. We applied the motions to each joint with pre-calculated algorithm on robot movement. And we observed the robot walking according to that motions.

In this study, we studied the method of motion construction according to the gait of each robot and measured the torque applied to joint. Also we verified that the developed wireless servo motor by ZigBee sensor network offers easier control and better circuit configuration.

[Table.1] comparison of wired and wireless type

\begin{tabular}{|c|c|c|}
\hline Type & merit & demerit \\
\hline wired & $\begin{array}{c}\text { - Parallel control is able } \\
\text {-Series motor connection } \\
\text { is able }\end{array}$ & $\begin{array}{c}\text {-Noise generation from wires } \\
\text { - Malfunction by rubbing } \\
\text { wires while walking } \\
\text {-Complexity of control wires }\end{array}$ \\
\hline wireless & $\begin{array}{c}\text {-Control of wireless } \\
\text { response } \\
\text {-Minimization of noise } \\
\text { from wires } \\
\text {-Simplification of wires } \\
\text {-Convenient transmission } \\
\text { of data }\end{array}$ & $\begin{array}{c}\text { - Weakness of wave strength } \\
\text { - High manufacturing cost }\end{array}$ \\
\hline
\end{tabular}

\subsection{Models of walking robot}

Fig. 1. shows the structure of two-legged walking robot which was constructed in this study.

(a)-coordinate indicates the criteria coordinate system.

(b)-coordinate indicates the fixed coordinate system of robot.

(c)-coordinate indicates the vector of force applied to each joint of robot. Setting of the path against the existing coordinate system for each leg is shown as below. The vectors connected to the body and right leg(R-Leg) from the criteria coordinate system(a) are expressed as $\mathrm{R}$ and $\mathrm{J}$ respectively, the position of tiptoe of right leg from body coordinate system as $\mathrm{P}$, and walking trace of leg as J. Vector of force applied to (c)-coordinate system is expressed as Kc.

(b)

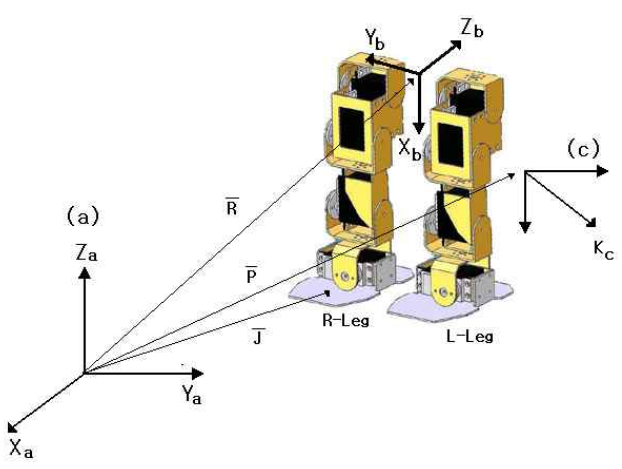

Fig. 1. Structure of walking robot and coordinate system

\subsection{Walking steps of robot}

There are two phases in robot gait same as human style walking has.

First, Single support phase ; one foot moves along the walking trace while the other one is supporting the body.

Second, Double support phase ; two feet support the body together after the foot moving along the trajectory of leg reaches the ground. Human gait is progressed repeating that the one foot moves along the trajectory of leg, and then two feet exchange their roles. Generally when human walks, toe or heel reaches the ground in advance to reduce the impact of contact and repulsive power between the ground and foot, but this kind of walking is excluded in this study, and we experimented that one leg moves the center of gravity generated from walking while the other leg is supporting the body. At this time we performed the gait with checking the gravity center on sole by using the pressure sensor attached to it. 


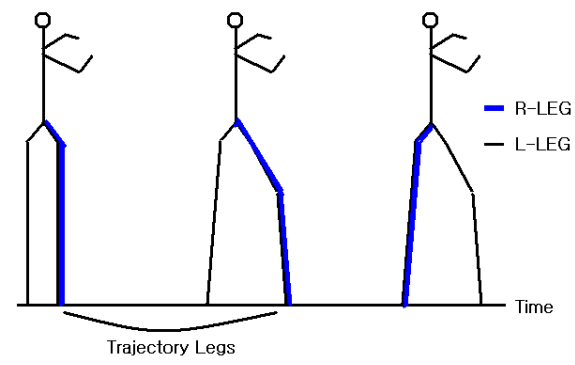

Fig. 2. Walking step of two-legged robot

\section{Design and Development}

This study describes the wireless digital servo motor developed for experimental walking of two-legged robot and system construction of human robot and hardware function.

\subsection{Construction of wireless digital servo motor}

The servo motor used for robot joints in this study is the wireless digital servo motor and was designed to use ZigBee sensor network, and is equipped with software of ZigBee Stack. The structure of ZigBee Stack is shown in Fig. 3. On-chip solution of MG2400 is used inside of servo motor, and library where codes are composed on the basis of the standard of IEEE 802.15.4. Zigbee Stack has two kinds of method (KVP and MSG) to transmit the data, and the difference between two methods is whether the type of data is designated or not. In the case of using MSG type, all the data as many as designated packet can be received if length in the data packet sent properly. In this study, we performed the emulation of the communication by MSG type as if the user is performing the serial communications. Wireless digital servo motor can be controlled by serial communication interface that communicates with data packet at $9600 \mathrm{bps}$.

[Table.2] Serial Data Packet Format

\begin{tabular}{|c|c|c|c|c|c|}
\hline $\begin{array}{c}\text { STX } \\
\text { (0xAA })\end{array}$ & Length & Command & Contents & $\begin{array}{c}\text { Check } \\
\text { SUM }\end{array}$ & $\begin{array}{c}\text { ETX } \\
(0 x 55)\end{array}$ \\
\hline 1 Byte & 1 Byte & 1 Byte & Variables & 1Byte & 1 Byte \\
\hline
\end{tabular}

DC motor, Metal speed reducer, and Rotary sensor to control the rotating direction and position of motor are equipped inside of actuator. Also ZigBee stack is constructed inside of the actuator to perform sensor network inside of ZigBee wireless module, and the network between actuators and main control system performs the control.

\section{$\square$ Zigbee Stack structure applied}

ZigBee stack is designed to port ZigBee stack software of sensor network to ZigBee sensor network module. Fig. 3 shows the basic structure of ZigBee stack.

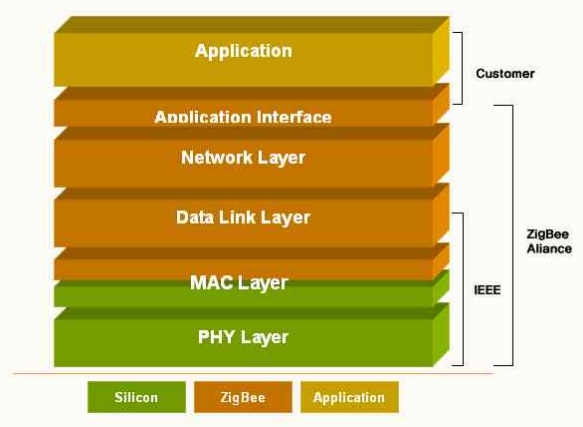

Fig.3. Structure of ZigBee Stack

Fig. 3 shows the structure of ZigBee stack software used for experiment in this study. This was applied with basic structure of ZigBee Alliance which is consisted of IEEE of PHY Layer, MAC Layer, Data Link Layer, and interface part for communications with APP to control Network layer and servo motor. APS and APP parts are consisted of APSDE and APSME, and APPlication part performs the control PID to control servo motor.

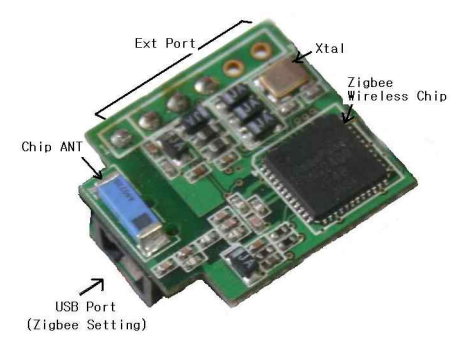

Fig. 4. Inside of ZigBee Sensor Network Board 
$\square$ Structure of Wireless digital servo motor

The following picture shows the appearance and inside of the developed servo motor. Digital servo motor is equipped with motor drive, rotary sensor for position control, and metal gear as speed reducer.

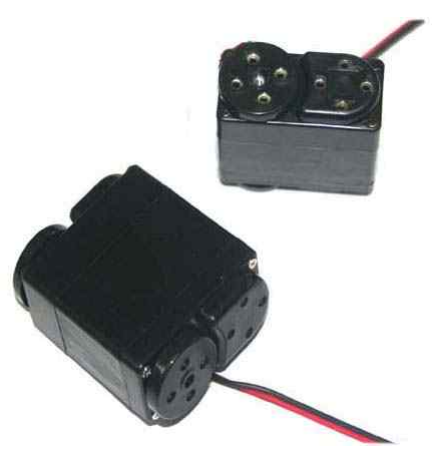

Fig. 5. Appearance of wireless digital servo motor

\subsection{Construction of Human Robot System}

This two-legged walking robot is constructed with wireless digital servo motor, designed to have 16 Degree Of Freedom joint structure, and wireless digital servo actuator is used for each joint. ATmega128 of ATmel as main unit, RISC type 8bit micro controller, is used to control the robot. This control unit is equipped with 2 -channel serial communication port. One port is used as a remote wireless transmission port to control each joint and the other one communicates with $\mathrm{PC}$ to control the robot and is used as a remote wireless port for simulation. And PT, ADC port, is used to receives the analog output signal from FSR pressure sensor attached to the robot sole.

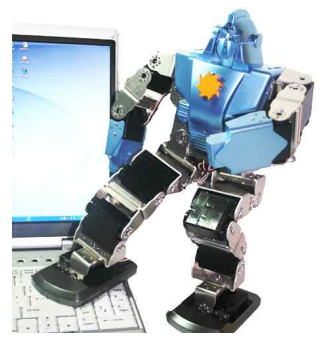

Fig. 6. Appearance of walking robot

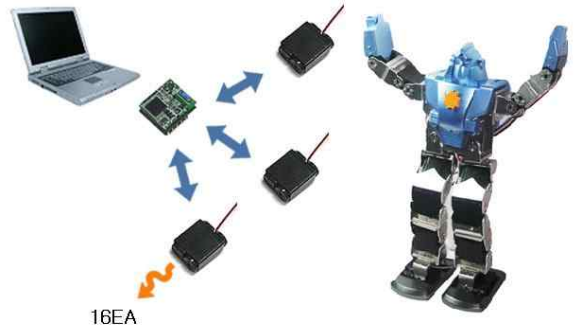

Fig. 7. Construction of robot system

The transmission of commands to control the angle of wireless digital servo motor placed at the robot joints made it possible to control the multi joints on real time, and thus, with this construction, the robot gait can be performed easily without wire for data transmission.

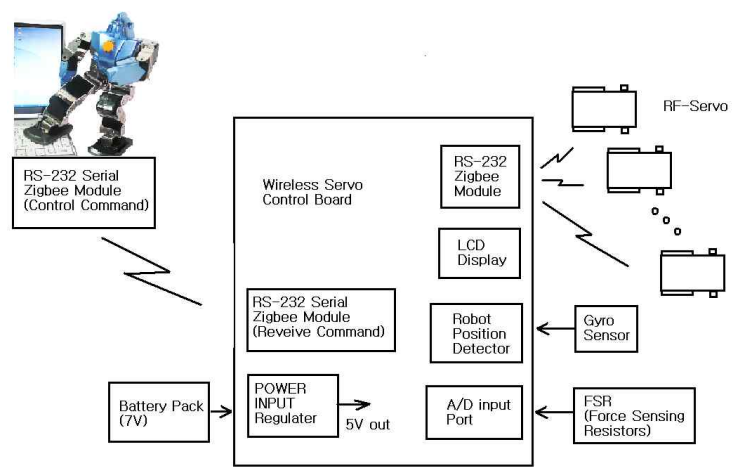

Fig.8. Construction of robot system

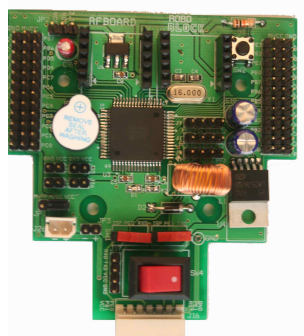

Fig.9. Control Board for 2-legged walking robot 


\section{Experiment}

We tested the torque of wireless servo motor used for Humanoid robot and conducted an experiment on the angle of conversion according to each motion.

\subsection{Experiment of wireless control servo}

We connected 16 Zigbee wireless control servo actuators and conducted an experiment on the motion control with wireless transmission of the signals from the central control unit. The following picture shows the central control unit of the actuator which was used in the experiment.

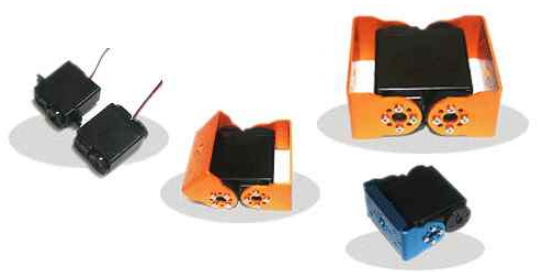

Fig.10. Actuator used for 2-legged walking robot

The force applied to actuator varies according to the weight of object, and the force is calculated as shown in the formula below.

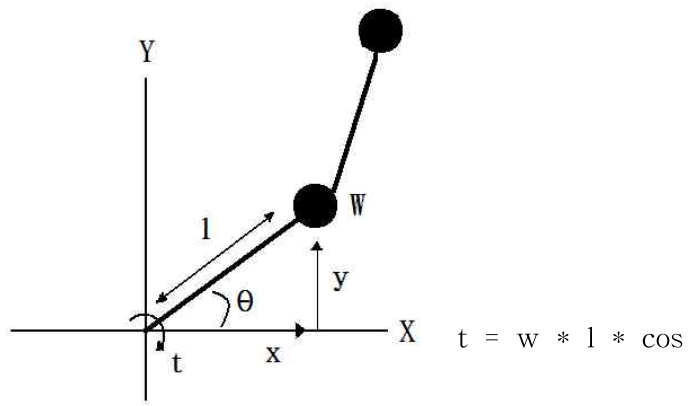

$\Theta(\mathrm{t}=$ torque, $\mathrm{w}=$ weight, $1=$ length $)$

Fig.11. Force of actuator joints per weight

The figure shows the angle against the changing amount. Value $t$ will be changed according to each change as well as the distance between axises of motors or other axises.

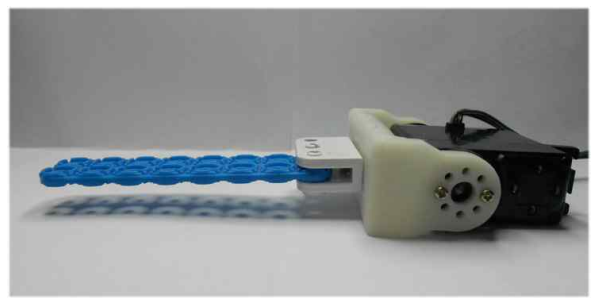

(a) Position 2

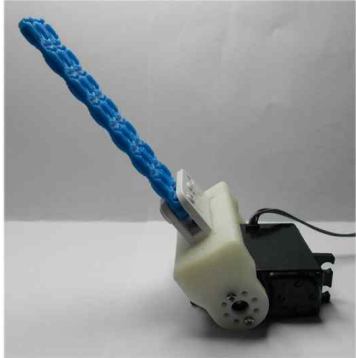

(b) Position 3

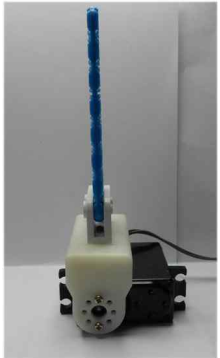

(c) Position 1

\section{Fig.12. Position according to change of Weight(W)}

We made a operating program to conduct an experiment on the changing amount according to each weight, and measured the changing amount, applying remote control signal.

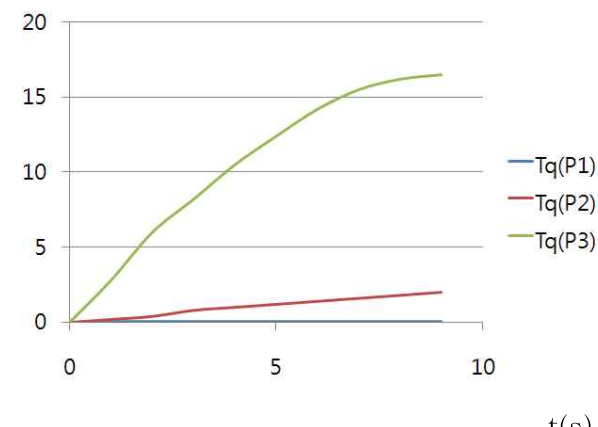

Tq(P1) : Torque of Position 1 [Kg.cm] $\mathrm{Tq}(\mathrm{P} 2):$ Torque of Position 2 [Kg.cm] $\mathrm{Tq}(\mathrm{P} 3)$ : Torque of Position 3 [Kg.cm]

Fig.13. Displacement according to changing amount of weight(W) 
3.2 Measurement of changing amount according to walking motion

We constructed the human leg style joints with wireless digital servo actuator, and conducted an experiment on each motion performing numeric modeling. The following figure shows the changing amount according to the axis of rotation and each weight, and shows the changing amount between standing and sitting motion.
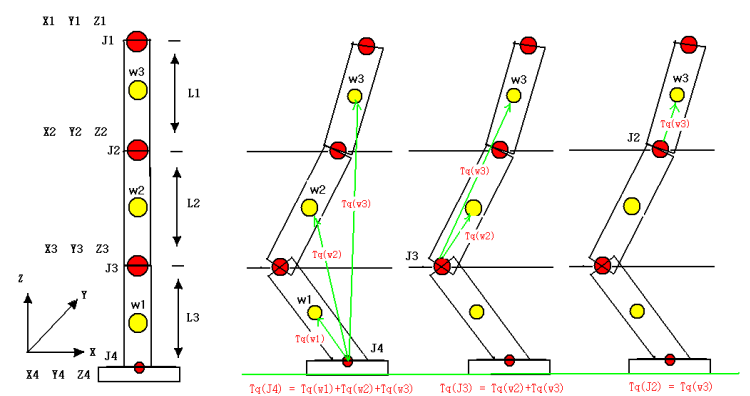

Joint 4 Torque : $\mathrm{Tq}(\mathrm{J} 4)=\mathrm{Tq}(\mathrm{w} 1)+\mathrm{Tq}(\mathrm{w} 2)+\mathrm{Tq}(\mathrm{w} 3)$

Joint 3, 2 Torque: $\mathrm{Tq}(\mathrm{J} 3)=\mathrm{Tq}(\mathrm{w} 2)+\mathrm{Tq}(\mathrm{w} 3) / \mathrm{Tq}(\mathrm{J} 2)=\mathrm{Tq}(\mathrm{w} 3)$

Fig.14. Displacement of 2-legged walking robot

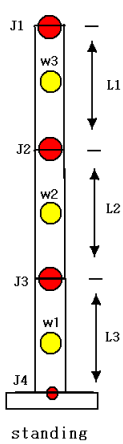

(1)

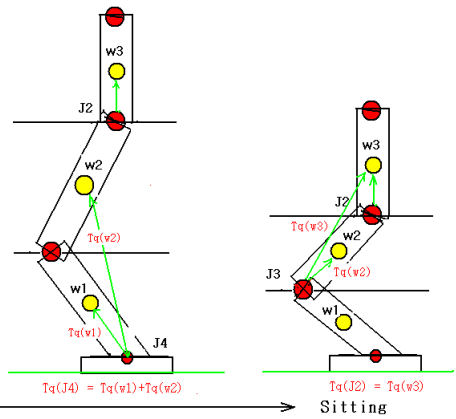

(3)
Fig. 15. Displacement between standing and sitting motion

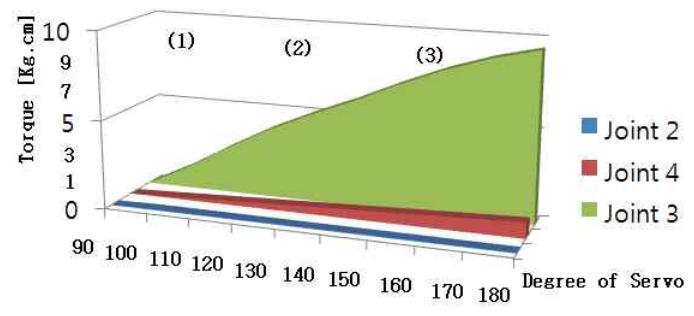

Fig. 16. Displacement of sitting motion

We measured the changing amount according to the change of weight while walking. The following figure shows the change of leg joint during the gait. We measured the change of one leg since the change of right and left leg is same.

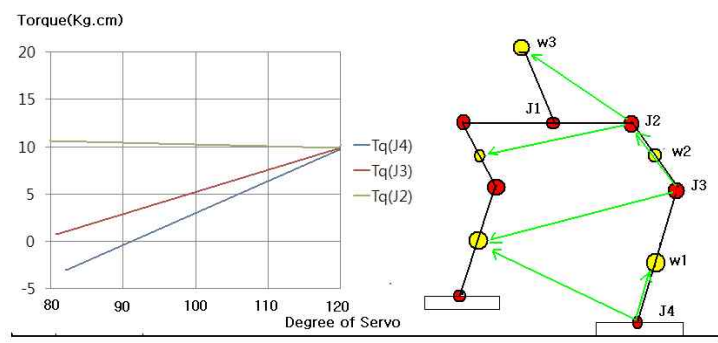

Fig. 17. Changing amount while walking

The Fig.17 shows the change according to each motion. We could perform effective walking motion by giving operating signals according to the changing amount. Also we acknowledged that efficient walking can be obtained by controlling the walking motion of robot according to the pressure change of FSR sensor attached to the sole.

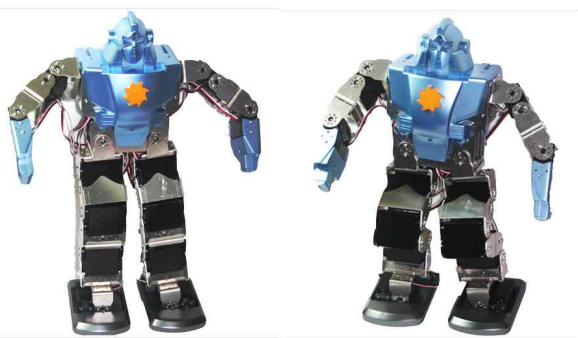

Fig. 18. Walking motion of 2-legged walking robot 
We extracted the trajectory of the gravity center from the trajectory of motion according to the strides of two-legged walking robot, and measured the torque change of ankle according to each motion.

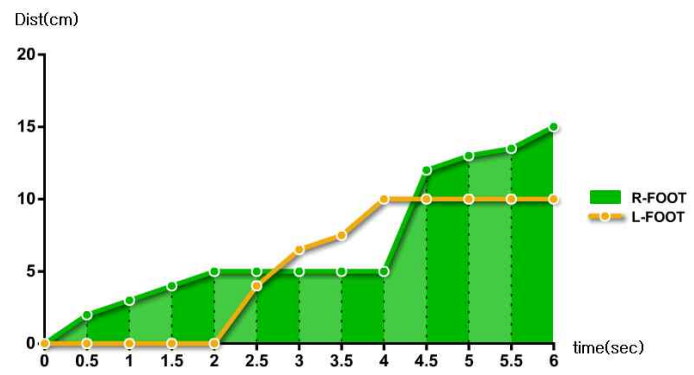

Fig. 19. Displacement according to strides

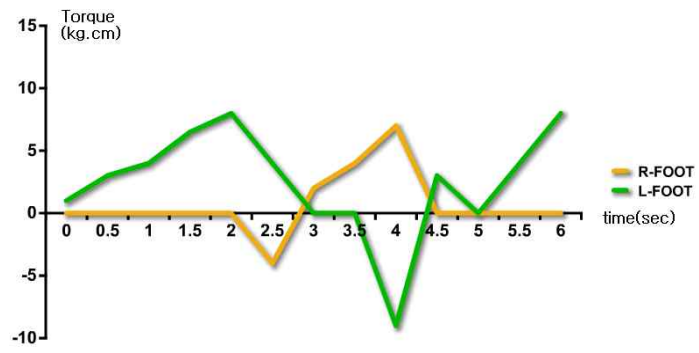

Fig. 20. Torque change of ankle

\subsection{Experiment of two-legged walking robot}

We developed the control environment of $\mathrm{PC}$ so as to experiment a simulation and motion for robot gait, and designed the robot to be controlled by the developed environment, main control unit, and wireless communication, and download to robot can be done. Construction of data for control is as below.

1) All the data on robot motions are treated as numeral row as below.

98, 86, 98, 92,102, 50, 95,100, 91, 94, 90, 95, 98, $90,130,95,101,10,50$
2) Classification of data numeral row

\begin{tabular}{|c|c|c|c|c|c|c|}
\hline Motor0 & Motor1 & $\ldots \ldots$ & \begin{tabular}{|c} 
Motor 1 \\
5 \\
\end{tabular} & \begin{tabular}{|c|} 
Motor1 \\
6 \\
\end{tabular} & Division & Delay \\
\hline 98 & 86 & & 95 & 101 & 10 & 50 \\
\hline
\end{tabular}

(1) Motor0 Motor16 : Position value of each motor

(2) Division : Resolution of the motor position prior to robot motion to the position moved to this value. The higher the value, the smoother the rotation, but the slower the speed is.

(3) Delay : Delay in moving within each value of the resolved motor position. The higher the value, the slower the speed is.

We checked the movement of robot by transmission of the control values of robot to the control program at PC. Fig.21 shows remote control program which is able to control and teach gait of two-legged walking robot.

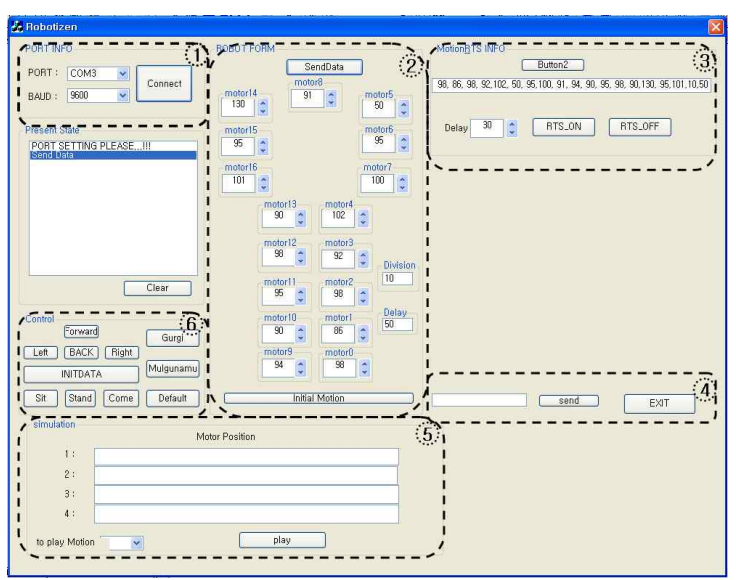

Fig. 21. Interface of robot

Construction of interface is

1) Port Info : Function to set, connect, and release the communication port and Baud.

(1) PORT : Port setting

(2) BAUD : Baud setting

(3) Connect : Connect and release

2) ROBOT FORM : Function to set the position, speed, and smoothness of each motor. 
(1) SendData : Transmit data to robot. The numeral row is additionally indicated on Edit window of MotionRTS INFO.

(2) Initial Motion : As basic pose, set each data value.

3) MotionRTS INFO : Indicate numeral row and set ROBOT FORM with numeral row.

(1) Button2 : Set data numeral row as ROBOT FORM

4) Serial Communication Region : Send numeral row on Edit window to robot directly.

5) Simulation : Region to simulate the robot motion, and simulation up to 4step is able, and data numeral row comes into Edit window. We can copy the data numeral row of MotionRTS INFO for use.

(1) to play motion : Determine which Edit window simulation is up to.

(2) play : Simulation

6) Control : Perform the pre-reserved motion by pressing each button.

\section{Conclusions and Future Research}

In this study, we constructed a two-legged walking robot with wireless digital servo actuator to which ZigBee sensor network technology is applied, and performed the control of each joint of robot by constructing ZigBee sensor network. And we effectively controlled the robot with remote control via control unit, and developed remote control teaching program to make robot gait effective by simulation of the values on robot motion at PC. Also we solved the noisy problems caused by the existing joint control system and improved the matters of inconvenience caused by expanding wires. Each joint is equipped with ZigBee sensor network module, and thus, we could conduct the experiment on the effective joint control. We attached the pressure sensor to the sole of robot to ensure stable walking. And we studied the method of motion construction which is according to robot gait, and could expect more effective and efficient control when this method is actually applied to Humanoid robot.

\section{References}

[1] J. Ziegler and W. Banzhaf, "Evolution of Robot Leg Movements in a Physical Simulation" in Proceedings of the Fourth Intermational Conference on Climbing and Walking Robots, CLA-WAR, 2001

[2] A. Boeing and T Braunl, "Evolving Splines: An alternative locomotion controller for a bipedal robot" in proceedings of the Seventh International Conference on Control Automation, Robotics and Vision (ICARCV 2002), 2002

[3] G. S. Hornby, S.Takamura, J. Yokono, O. Hanagata, M. Fujita and J. Pollack, "Evolution of Controllers from a High-Level Simulator to a High DOF Robot." in Evolvable Systems: from biology to hardware : Proceedings of the third international conference (ICES200), 2000.

[4] James Kuffner, Koichi Nishiwaki, Satoshi Kagami, Masayuki Inaba, "Motion Planning for Humanoid Robots. In Proc. 11th Int'l Symp. of robotics Research (ISRR2003)

[5] Dae Seob Shin, Hyeongcheol Lee, "A Study on the Actuator for Robot Control Using Wireless Zigbee Sensor Networks". Journal of IKEEE, Korea, 2011.

[6] Collins, S. H. Ruina, A.(2005) A bipedal walking robot with efficient and human-like gait. In Proc. IEEE Int, Conf. Robotics and Automation, Barcelona, Spain, In Press.

[7] Yildirim Hurmuzlu, Frank Genot, Bernard Brogliato "modeling, stability and control of biped robots- a general framework", ELSEIVER, France 2004.

[8] Jacky Baltes and Patrick Lam- "Design of walking gaits for Tao-pie-pie, a small humanoid robot", In Advanced Robotics, 18(7): 713-716, 2004. 


\section{BIOGRAPHY}

\section{SHIN DAE SEOB (Student Member)}

1996: BS degree in Electronics
Engineering ,Howon University.
1998: MS degree in Electronics
Engineering, Inha University.
Present: Ph. D. Course in
Electrical and Biomedical
Engineering, Hanyang University

$<$ Research Interests> Image processing, neural network, Adaptive control, Signal Processing, Embedded Control, Rehabilitation robots.

\section{Hyeongcheol Lee (Non Member)}

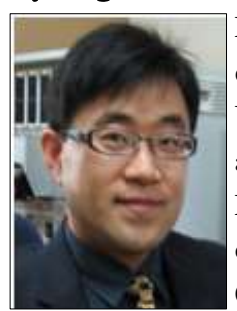

Received the B.S. and M.S. degrees from Seoul National University, Seoul, Korea, in 1988 and 1990, respectively, and the Ph.D. degree from the University of California at Berkeley, in 1997. Currently, he is a Professor with the Division of Electrical and Biomedical Engineering of Hanyang University, Seoul, Korea. $<$ Research interests $>$ Adaptive and nonlinear control, embedded systems, and applications to robotics and vehicle controls. 